\title{
Thermal-Diffusivity Measurements of Conductive Composites Based on EVA Copolymer Filled With Expanded and Unexpanded Graphite
}

\author{
I. H. Tavman - A. Turgut • H. M. da Fonseca • \\ H. R. B. Orlande - R. M. Cotta • M. Magalhaes
}

Received: 24 December 2010 / Accepted: 8 June 2012

(C) Springer Science+Business Media, LLC 2012

\begin{abstract}
In this research, the thermal diffusivity of composites based on ethylenevinyl acetate (EVA) copolymer filled with two kinds of reinforcement graphite materials was investigated. The reinforcement graphite fillers were untreated natural graphite (UG) and expanded graphite (EG). Composite samples up to $29.3 \%$ graphite particle volumetric concentrations ( $50 \%$ mass concentration) were prepared by the meltmixing process in a Brabender Plasticorder. Upon mixing, the EG exfoliates in these films having nanosized thicknesses as evidenced by TEM micrographs. Thus, the thermal diffusivity and electrical conductivity of composites based on the ethylene-vinyl acetate matrix filled with nanostructuralized expanded graphite and standard, microsized graphite were investigated. From the experimental results it was deduced that the electrical conductivity was not only a function of filler concentration, but also strongly dependent on the graphite structure. The percolation concentration of the filler was found to be (15 to 17) vol\% for micro-sized natural graphite, whereas the percolation concentration of the filler in nanocomposites filled with expanded graphite was much lower, about (5 to 6 ) vol\%. The electrical conductivity of nanocomposites was also much higher than the electrical conductivity of composites filled with micro-sized filler at similar concentrations. Similarly, the values of the thermal diffusivity for the nanocomposites, EG-filled EVA, were significantly higher than the thermal diffusivity of the composites filled with micro-sized filler, UG-filled EVA, at similar concentrations. For $29.3 \%$ graphite particle volumetric concentrations, the thermal diffusivity
\end{abstract}

\footnotetext{
I. H. Tavman $(\varangle) \cdot$ A. Turgut

Department of Mechanical Engineering, Dokuz Eylul University, 35100 Bornova, Izmir, Turkey e-mail: ismail.tavman@deu.edu.tr
}

H. M. da Fonseca · H. R. B. Orlande - R. M. Cotta · M. Magalhaes

COPPE/UFRJ Department of Mechanical Engineering, Federal University of Rio de Janeiro-UFRJ,

Rio de Janeiro 21941-972, Brazil 
was $8.23 \times 10^{-7} \mathrm{~m}^{2} \cdot \mathrm{s}^{-1}$ for EG-filled EVA and $6.14 \times 10^{-7} \mathrm{~m}^{2} \cdot \mathrm{s}^{-1}$ for UG-filled EVA. The thermal diffusivity was measured by the flash method.

Keywords Conductive nanocomposites - Electrical conductivity $\cdot$ EVA . Expanded graphite $\cdot$ Unexpanded graphite $\cdot$ Flash method $\cdot$ Thermal diffusivity

\section{Introduction}

Polymeric materials are limited in their applications because of their inherent low thermal conductivity and diffusivity, low thermal stability, high electrical resistivity, and ductile mechanical properties. However, polymers are easily processed to give them the required forms, and they are inexpensive and have high strength-to-weight ratios. With the addition of micro- and nano-meter size particles or fibers into a polymer matrix, the properties may be drastically enhanced and become suitable for the particular use intended for the material. For this, it is important to choose the right kind of polymer and filler as well as the concentration of the filler and processing method. The shape and size of the filler material are extremely important, in general micro-sized additions weakens the mechanical properties of polymers; whereas, for the same type, the addition of nano-sized fillers causes an improvement in mechanical properties. For a given particulate volume fraction, the composite strength increases with decreasing particle size. Smaller particles have a higher total surface area for a given particle loading. This indicates that the strength increases with increasing surface area of the filled particles through a more efficient stress-transfer mechanism [1]. In brief, the particle size clearly has a significant effect on the strength of particulate-filled polymer composites, which generally increases with decreasing size. In order to make polymeric materials electrically and thermally conductive, metals, metal oxides, and carbon fillers are added to the matrix material. Among a number of techniques that are used in the preparation of nanocomposites, melt mixing is the most widely used because of the ease of preparation and it's less time consuming than the other processes. Numerous carbon additives have been used to enhance the properties of a pure polymer, the most popular being carbon blacks, natural graphite, expanded graphite, carbon nanotubes, and carbon nanofiber. Graphite is a naturally occurring, or synthetically produced, crystalline form of carbon that is highly conductive (with an electrical conductivity of $104 \mathrm{~S} \cdot \mathrm{cm}^{-1}$ at ambient temperature).

Recently, polymer-based nanocomposites reinforced with expanded graphite (EG) have shown substantial improvements in mechanical, electrical conductivity, thermal conductivity, and barrier properties over the pure polymer. The explanation for this lies in the fact that the natural graphite has a sheet-like structure where the atoms are strongly bonded on a hexagonal plane but weakly bonded normal to that plane. From these sheets, if layers could be separated down to a nanometer thickness, they would form high aspect ratio (200 to 1500 ) and high modulus ( $\sim 1 \mathrm{TPa}$ ) graphite nanosheets [2]. Then, the graphite nanosheets could have an enormous surface area (up to $2630 \mathrm{~m}^{2} \cdot \mathrm{g}^{-1}$ ) considering both sides of the sheets are accessible [3]. Therefore, the dispersion of such nanosheets in a matrix will play a key role in the improvement of both physical and mechanical properties of the resultant nanocomposite. This can be 
achieved by a combination of both synthesis and processing techniques that produces complete exfoliation and good dispersion of graphite particles in the matrix.

The synthesis of EG and the formation of graphite nanosheets are well-documented in the literature [2-5]. First, the natural graphite is converted to intercalated or expandable graphite through chemical oxidation in the presence of concentrated $\mathrm{H}_{2} \mathrm{SO}_{4}$ and $\mathrm{HNO}_{3}$ acid. Then, the EG is obtained by rapid expansion and exfoliation of expandable graphite in a furnace above $600{ }^{\circ} \mathrm{C}$. A good number of studies have been conducted on EG-reinforced conductive polymer nanocomposites [4,6-11]. Most of the investigations have been carried out on thermoplastics such as polystyrene [4], PMMA [6,7], nylon-6 [8], and polypropylene [9]. These nanocomposites were prepared via in situ polymerization, solution compounding, or melt mixing and their mechanical, electrical, and thermophysical properties of the resultant nanocomposites were measured.

\section{Experimental}

\subsection{Materials}

In this study an ethylene-vinyl acetate copolymer (EVA) containing 14 mass \% of vinyl acetate (VA), Miravithenß D 14010 V supplied by Leuna Polymer GmbH, Germany was used as the matrix material. Its melt flow index is $9.8 \mathrm{~g} / 10 \mathrm{~min}\left(190{ }^{\circ} \mathrm{C} / 2.16 \mathrm{~kg}\right)$. The filler materials were EG having originally a size in the range of (5 to 6 ) $\mu \mathrm{m}$ in length and untreated graphite (UG) having a size from $20 \mu \mathrm{m}$ to $25 \mu \mathrm{m}$. Most of the particles have an aspect ratio between 20 and 250. Figure 1 shows an SEM image of EG.

\subsection{Preparation of Composites}

EVA — graphite mixtures were prepared in a Brabender Plasticorder PLE 331 internal mixer at $150{ }^{\circ} \mathrm{C}$ for a total mixing time of $10 \mathrm{~min}$, the mixing chamber capacity being

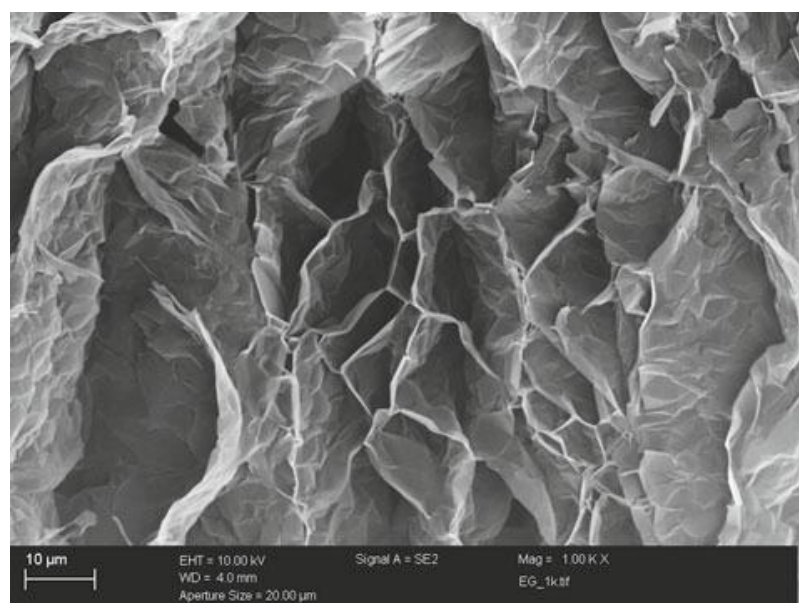

Fig. 1 SEM image of EG 

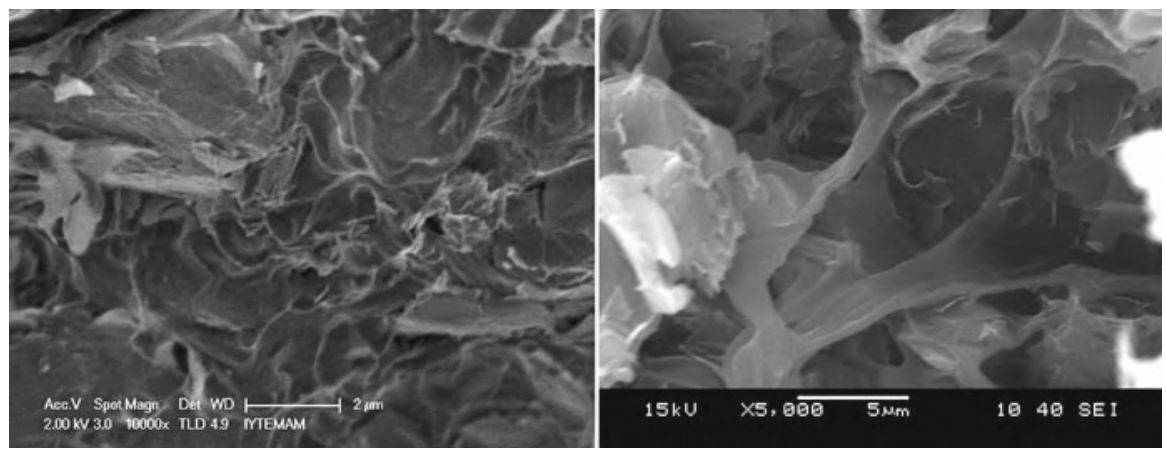

Fig. 2 SEM images of EVA filled with $15 \%$ by mass of EG nanocomposite

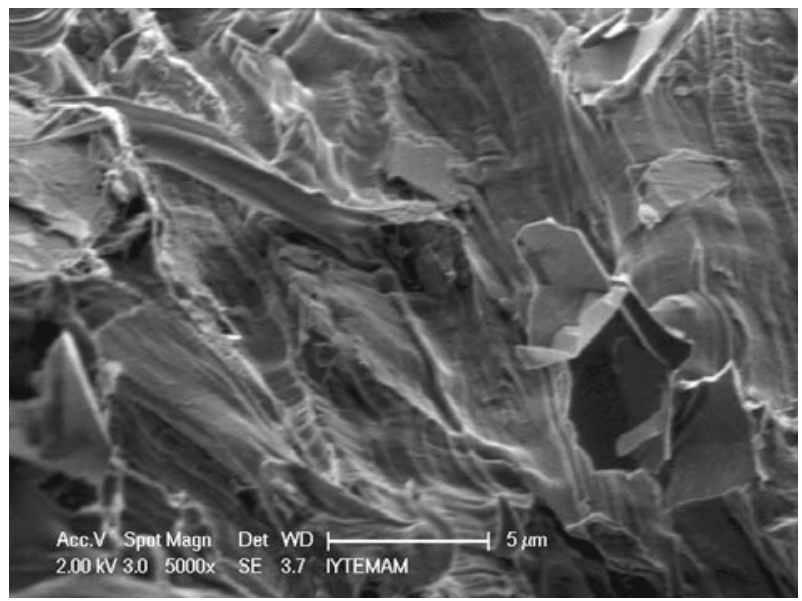

Fig. 3 SEM image of EVA filled with $15 \%$ by mass of unexpanded graphite (UG) composite

$30 \mathrm{~mL}$. The rotors turned at $35 \mathrm{rpm}$ in a counter-rotating fashion with a speed ratio of 1.1. After $10 \mathrm{~min}$, the mixing chamber of the Brabender apparatus is opened and the resulting mixture is taken out, then after passing through rollers, the mixture is solidified. The resultant mixture in then put in a compression molding die and compressed in a compression molding press at $120^{\circ} \mathrm{C}$, under $40 \mathrm{kP}$ pressure for one minute to obtain samples in the form of sheets for electrical-conductivity and thermal-diffusivity measurements. During this mixing process, the EG exfoliates in nanosized graphite layers causing nanocomposites of improved physical properties. Figure 2 shows the SEM images of EVA filled with 15 mass\% of an EG nanocomposite. Figure 3 shows the SEM image of EVA filled with 15 mass\% of an unexpanded graphite (UG) composite. The SEM images of the composites were taken at the breaking point of tensile test specimens.

The effects of mixing time and temperature on electrical and mechanical properties have been studied, and the mixing time of $10 \mathrm{~min}$ and temperature of $180{ }^{\circ} \mathrm{C}$ were found to be optimal values. Longer mixing times decreased the mechanical strength of the composites. The homogeneity of the samples has been determined by 
different mechanical, thermal, and electrical tests. Tensile strength tests, with specimens, taken at different locations did not show differences in the test values. Also, electrical-conductivity tests with samples taken at different locations did not show appreciable differences. Upon chaotic mixing in the Brabender apparatus, EG particles exfoliate and form graphite nanosheets, which possess a huge surface area and high aspect ratio. Although the EG/EVA nanocomposites are anisotropic at the nanolevel, they are isotropic for macro-level samples, as there is no preferred orientation due to the process of preparation.

\subsection{Flash Method}

In 1961, Parker et al. [10] published their pioneering work on the development of a method for determination of the thermal diffusivity of solid materials. The method, well-described in the literature [11-14], is elegant as it reduces a difficult thermal measurement to a much more manageable time measurement. However, the technical level of the flash method needs development in order to decrease the uncertainty in thermal-diffusivity measurements. For this purpose, a number of studies have been conducted in order to correct for the pulse length [15-17], and the radiative heat losses [18,19].

In this method, a uniform high intensity heat pulse of short duration compared to the transient time through the sample is incident on the front face $(x=0)$ of a disc- or square-shaped sample with a thickness of a few millimeters. The temperature rise on the rear face $(x=e)$ is recorded, as illustrated in Fig. 4. The sample is coated with absorbing black paint if the sample is transparent to the heat pulse. If two dimensionless parameters $V$ and $\omega$ are defined by

$$
V(e, t)=\frac{T(e, t)}{T_{\mathrm{M}}}
$$

Fig. 4 Dimensionless temperature history at the rear face

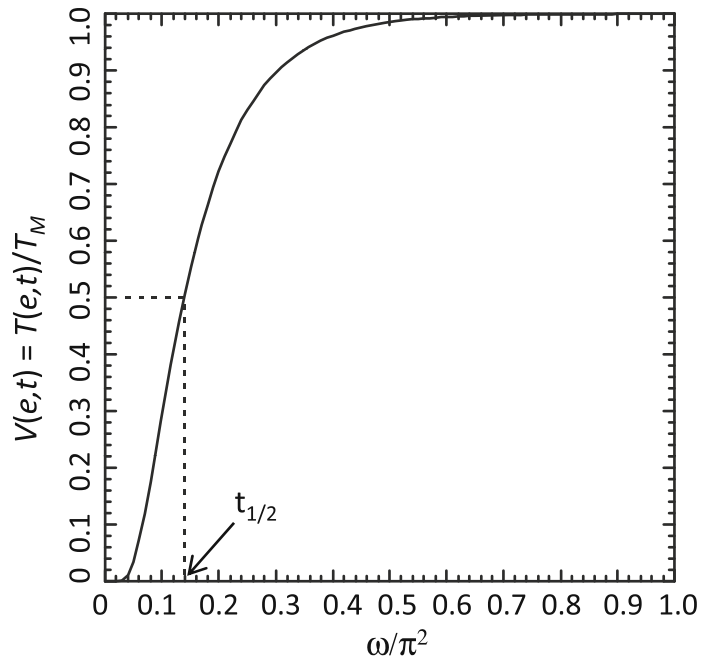




$$
\omega=\frac{\pi^{2} a t}{e^{2}}
$$

where $T(e, t)$ and $T_{\mathrm{M}}$ are the instantaneous and maximum temperature increases of the rear face; $e$ is the sample thickness; $t$ is the time, and $a$ is the thermal diffusivity.

If no heat losses occur, the dimensionless temperature increase on the rear face is given by

$$
V(e, t)=1+2 \sum_{n=1}^{\infty}(-1)^{n} \exp \left(-n^{2} \omega\right)
$$

It is a common practice to employ the half-maximum temperature rise time $t_{1 / 2}$ for which $\omega$ is equal to 1.38 ; hence, the thermal diffusivity $(a)$ can be calculated using the following expression:

$$
a=\frac{1.38 e^{2}}{\pi^{2} t_{1 / 2}}
$$

In this study, we used the flash apparatus Netzsch Nanoflash LFA 447/1. The LFA $447 / 1$ is a tabletop instrument that works with a high power xenon-flash lamp as heat pulse source. The temperature range for measurements can be adjusted from room temperature to $200{ }^{\circ} \mathrm{C}$; four samples can be charged in the sample holder. An integrated furnace (heater) maintains the sample temperature stable during measurements. Figure 5 shows a schematic view of the instrument systems. A high-power xenon flash lamp is surrounded by a parabolic mirror (reflector). The xenon flash lamp supplies a pulse energy up to $10 \mathrm{~J}$, adjustable to the desired power by the measurement software. The pulse duration can also be adjusted by software to $(0.1,0.2$, or 0.4$) \mathrm{ms}$. The LFA $447 / 1$ uses an InSb infrared detector in the wavelength range of $2000 \mathrm{~nm}$ to $5000 \mathrm{~nm}$ and is cooled by liquid nitrogen. This allows a non-contact fast measurement of the temperature rise in the rear of the sample. The Netzsch-LFA apparatus has built-in software for calculating, by a curve-fitting method, the thermal diffusivity from the transient temperature history curve obtained at the rear face. The expected uncertainty for thermal-diffusivity measurements is (3 to 5) \% for most materials.

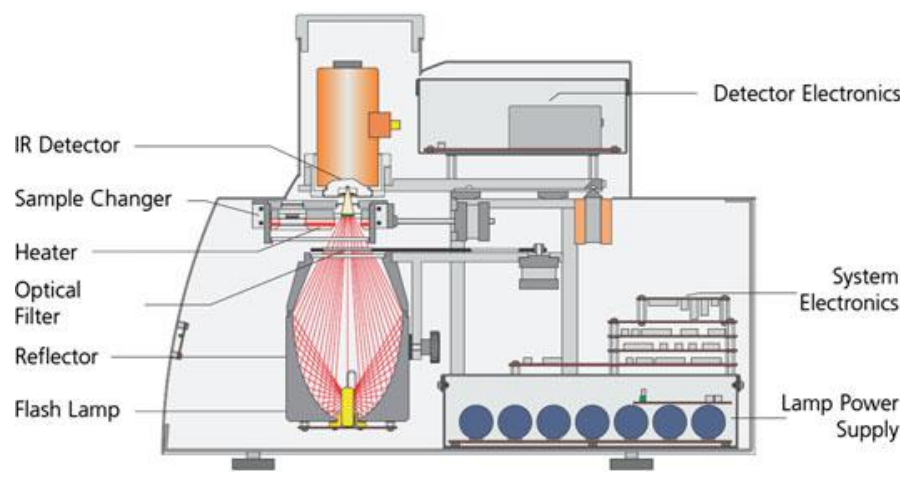

Fig. 5 Netzsch Nanoflash LFA 447/1 


\section{Results and Discussion}

\subsection{Electrical Conductivity}

The samples for electrical-conductivity measurements were square-shaped with a side length of $45 \mathrm{~mm}$ and a thickness of $4 \mathrm{~mm}$. Depending on the electrical conductivity of the samples, two methods were used for the measurement of the volumetric electrical conductivity. All the properties were measured at room temperature $\left(20^{\circ} \mathrm{C}\right.$ to $\left.25^{\circ} \mathrm{C}\right)$. For samples with an electrical conductivity lower then $10^{-5} \mathrm{~S} \cdot \mathrm{m}^{-1}$, the measurements were performed by a two-electrode system using only a Keithley 2400 source meter. In these cases, current-voltage characteristics were measured in a DC field. The specific electrical conductivity $(\sigma)$ can be expressed as follows:

$$
\sigma=\frac{1}{U} \frac{e}{A}
$$

where $I$ is the electrical current, $U$ is the voltage, $A$ is the area of electrodes, and $e$ is the thickness of the sample.

For samples with an electrical conductivity higher then $10^{-5} \mathrm{~S} \cdot \mathrm{m}^{-1}$, the measurements were performed by a four-electrode system (Van der Pauw) [20], using the combination of a Keithley 2400 high precision current source and Keithley 2182 nanovoltmeter. The four electrodes were positioned along a straight line, with spacing $s$. A current $(I)$ is passed through the sample via the outer probes (force probes), and the voltage $(U)$ is measured at the inner probes (sensing probes). The electrical resistivity $(\rho)$ is then obtained by the following equation:

$$
\rho=2 \pi F s R
$$

where $R$ is the resistance $(R=U / I)$ and $F$ is a correction factor depending on the sample geometry (sample thickness $(e)$ and distances from the edges of the sample). For $s \gg e, F$ can be expressed as

$$
F=\frac{e}{2(\ln 2) s}
$$

The electrical conductivity which is the inverse of the electrical resistivity can be calculated as

$$
\sigma=\frac{1}{\rho}
$$

A main advantage of four-probe measurements is the elimination of the contact resistance [21].

The variation of the electrical conductivity of both EVA-unexpanded graphite (UG) and EVA-EG composites is shown in Fig. 6 as a function of volumetric filler content. In the two cases the steep increase of the electrical conductivity was observed around a particular concentration of the filler. This concentration is called the percolation 


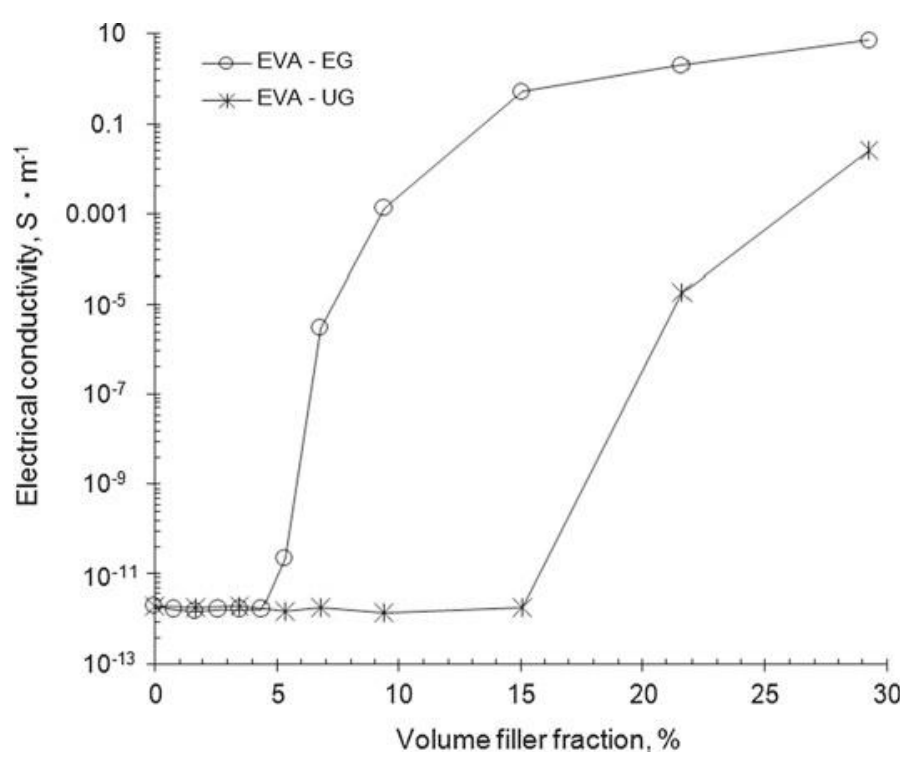

Fig. 6 Electrical conductivity of EVA-EG and EVA-UG composites versus volume filler fraction

concentration or percolation threshold. At this concentration, clusters of conductive particles are formed in a polymeric matrix and they represent a conductive path for a movement of electrons throughout the sample. It may be seen from Fig. 6 that the percolation threshold is (15 to 17) vol\% for micro-sized natural graphite-filled EVA and it is much higher compared with the percolation threshold of the EG-filled EVA nanocomposite, for which the percolation threshold is only (5 to 6 ) vol\%. The electrical conductivity of nanocomposites was also much higher than the electrical conductivity of composites filled with micro-sized filler at similar concentrations. The electrical conductivity for pure EVA was $1.99 \times 10^{-14} \mathrm{~S} \cdot \mathrm{cm}^{-1}$, whereas this value was $2.455 \times 10^{-4} \mathrm{~S} \cdot \mathrm{cm}^{-1}$ for 29.3 vol\% UG-filled EVA composites and $7.24 \times 10^{-2} \mathrm{~S} \cdot \mathrm{cm}^{-1}$ for 29.3 vol\% EG-filled EVA nanocomposites.

\subsection{Thermal Diffusivity}

The thermal diffusivity of the composites samples was measured by the flash technique at room temperature $\left(20^{\circ} \mathrm{C}\right.$ to $\left.25^{\circ} \mathrm{C}\right)$. The samples were square-shaped with a side length of $10 \mathrm{~mm}$ and a thickness of $4 \mathrm{~mm}$. For each filler concentration, four samples were prepared and five measurements were performed on each of these samples.

By analogy to the electrical-conductivity results, the thermal diffusivity increases as the filler content increases, Fig. 7. Uniform dispersion of the filler has high influence on the increase in thermal diffusivity as in the case of electrical conductivity. Since graphite is a good conductor of heat and electricity, an increase in graphite level will result in an increase in the electrical conductivity and thermal diffusivity of the EVA/graphite composites. 


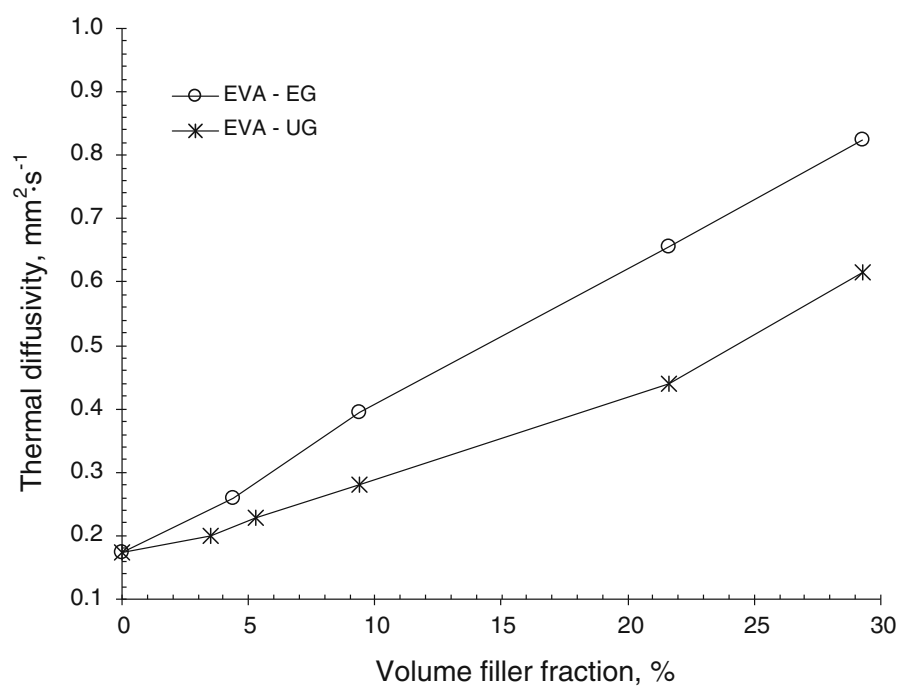

Fig. 7 Thermal diffusivity of EVA-EG and EVA-UG composites versus volume filler fraction

Figure 7 shows the thermal diffusivity of the exfoliated graphite-filled EVA versus the unexpanded graphite-filled EVA at various filler contents. As with the electrical conductivity, it can be seen that the thermal diffusivity of EG-filled EVA increases more rapidly than for unexpanded graphite-filled EVA composite. The maximum thermal diffusivity occurs at $29.3 \mathrm{vol} \%$ concentration of filler, this value is $8.23 \times 10^{-7} \mathrm{~m}^{2} \cdot \mathrm{s}^{-1}$ for EG-filled EVA and $6.14 \times 10^{-7} \mathrm{~m}^{2} \cdot \mathrm{s}^{-1}$ for UG-filled EVA which means an increase of 4.7 times for the EG/EVA nanocomposite, while the increase was only 3.5 times for the UG/EVA composite over that for pure EVA having a thermal diffusivity of $1.74 \times 10^{-7} \mathrm{~m}^{2} \cdot \mathrm{s}^{-1}$. This is due to the exfoliation of the graphite flake into sheets producing highly conductive material with a higher aspect ratio for EG than for typical natural graphite (UG).

\section{Conclusions}

In this study, we focused on comparing the electrical conductivity and thermal diffusivity of two different graphite-reinforced EVAs: one with UG and one with EG. The materials were mixed in a Brabender Plasticorder PLE 331 internal mixer at $150{ }^{\circ} \mathrm{C}$ for a total mixing time of $10 \mathrm{~min}$. Apparently, the EG system demonstrated better properties arising from a larger surface area and, as a result, a higher aspect ratio, compared to the UG system. The electrical conductivity for the composites showed a transition from an insulator to a conductor. Only (5 to 6) vol\% filler content was required to reach the percolation threshold for EG-containing EVA, whereas significantly higher ((15 to 17) vol\%) filler content was required for the UG system. Similarly, the incorporation of EG in EVA provided a significant improvement in thermal diffusivity compared with the unexpanded graphite in EVA. An understanding of the transition would allow future design for nanocomposites using EG fillers. As also noted in a recent 
study on electrical and thermal conductivities of ethylene-octene copolymer/expandable graphite composites [22], thermal diffusivity of the composites showed a trend of increment with addition of graphite filler, but no percolation threshold was observed. An understanding of the transition would allow future design for nanocomposites using EG fillers.

Acknowledgments This research was supported by the Scientific Support of the bilateral Project No. $107 \mathrm{M} 227$ of TUBITAK and SAS (Slovak Academy of Sciences).

\section{References}

1. S.Y. Fu, X.Q. Feng, B. Lauke, Y.W. Mai, Compos. Part B 39, 933 (2008)

2. L.T. Drzal, H. Fukushima, Polym. Prepr. 42, 42 (2001)

3. L.M. Viculis, J.J. Mack, R.B. Kaner, Science 299, 1361 (2003)

4. G. Chen, C. Wu, W. Weng, D. Wu, W. Yan, Polymer 44, 1781 (2003)

5. G. Chen, W. Weng, D. Wu, C. Wu, J. Lu, P. Wang, X. Chen, Carbon 42, 753 (2004)

6. W. Sheng, S.C. Wong, Compos. Sci. Technol. 63, 225 (2003)

7. W. Zheng, S.C. Wong, H.J. Sue, Polymer 73, 6767 (2002)

8. Y.X. Pan, Z.Z. Yu, Y.C. Ou, G.H. Hu, J. Polym. Sci., Part B 38, 1626 (2000)

9. J.W. Shen, X.M. Chen, W.Y. Huang, J. App. Polym. Sci. 88, 1864 (2003)

10. W. Parker, R. Jenkins, C. Buttler, G. Abott, J. Appl. Phys. 32, 1679 (1961)

11. R.D. Cowan, J. Appl. Phys. 34, 926 (1963)

12. K. Beedham, L.P. Dalrymple, Rev. Int. Hautes Temp. Refract. 7, 278 (1970)

13. A.B. Donaldson, J. Appl. Phys. 43, 4226 (1972)

14. L.M. Clark III, R.E. Taylor, J. Appl. Phys. 46, 714 (1975)

15. A. Degiovanni, Int. J. Heat Mass Transf. 30, 2199 (1987)

16. J. Cape, G. Lehman, J. Appl. Phys. 34, 1909 (1963)

17. T. Lechner, E. Hahne, Thermochim. Acta 218, 341 (1993)

18. L.M. Clark III, R. Taylor, J. Appl. Phys. 46, 714 (1975)

19. T. Baba, A. Ono, Meas. Sci. Technol. 12, 2046 (2001)

20. L.J. van der Pauw, Philips Res. Rep. 13, 1 (1958)

21. F.G. de Souza Jr., B.G. Soares, J.C. Pinto, Eur. Polym. J. 44, 3908 (2008)

22. P. Svoboda, R. Theravalappil, S. Poongavalappil, J. Vilcakova, D. Svobodova, P. Mokrejs, A. Blaha, Polym. Eng. Sci. 52, 1241 (2012) 